\title{
Comparative study of real-time HEV energy management strategies
}

\author{
Qi JIANG, Florence OSSART, Claude MARCHAND
}

\begin{abstract}
An adequate energy management strategy is the key to optimize hybrid electric vehicle fuel efficiency. Various real-time controls have been recently developed. As each study is performed in a specific context, a comparative analysis is critically needed to point out their pros and cons. The present paper proposes a comparison between three recent promising real-time strategies: adaptive equivalent consumption minimization strategy (A-ECMS), optimal control law (OCL) and stochastic dynamic programming (SDP). Two off-line algorithms are used as benchmark: Pontryagin's minimum principle and dynamic programming. Implementation and parameters setting issues are discussed for each strategy: a genetic algorithm is employed for A-ECMS calibration and SDP uses Markov chains for driving cycle prediction. The real-time strategies robustness is then evaluated over several types of driving cycles and a statistical analysis is conducted using random cycles generated by Markov process. Simulation results show that the original OCL requires modifications to achieve good performance on the considered powertrain model. A-ECMS reaches the best fuel saving performance when used with parameter sets adjusted to the type of driving conditions, while SDP better respects the charge sustaining constraint.
\end{abstract}

Index Terms-hybrid electric vehicle, real-time energy management, optimal control, Markov chain, A-ECMS, OCL, Pontryagin minimum principle, stochastic dynamic programming.

\section{INTRODUCTION}

$\mathrm{H}^{\prime}$ ybrid electric vehicles (HEVs) are widely considered as a potential alternative to the world's dependence on conventional vehicles. Thanks to fuel economy and emission control, HEVs contribute to the construction of an environment friendly transportation sector. HEVs possess an internal combustion engine (ICE) and at least one electric machine (EM) and a battery. The wheels can be powered either by the ICE or the EM or by both simultaneously. The addition of a battery-powered EM increases the total efficiency of HEVs. It allows the ICE to be more efficiently operated and the vehicle's kinetic energy to be recovered. However, the fuel saving and $\mathrm{CO}_{2}$ emissions reduction strongly depend on the embedded

Manuscript received July xx, 2016; revised xxxx xx, xxxx; accepted xxxx All three authors are with the Group of Electrical Engineering - Paris, 3, 11 rue Joliot-Curie, Plateau de Moulon F-91192 Gif-sur-Yvette, France (e-mail: qi.jiang@geeps.centralesupelec.fr; florence.ossart@geeps.centralesupelec.fr; claude.marchand@geeps.centralesupelec.fr)

Color versions of one or more of the figures in this paper are available online at http://ieeexplore.ieee.org.

Digital Object Identifier xxxx energy management strategy that defines the contribution of the two power sources (ICE and EM) in fulfilling a given power demand. The design of robust real-time optimization algorithms remains a challenge.

There is a very rich literature on the subject and many energy management strategies have been developed since the 90s. They can be divided into four approaches: rule-based strategies that are generally used on today's marketed HEVs [1], instantaneous optimization of an equivalent fuel consumption accounting for electricity flux [2][3][4][5][6][7], global optimization based on the dynamic programming [8][9][10][11][12][13][14][15] and convex optimization that was recently developed by modeling each component of the powertrain as convex equations [16]. Each strategy is shown to allow a significant reduction of fuel consumption and claimed to have better performances than others. However, the different studies are performed in their own specific context, making it difficult to evaluate the results.

The present paper proposes a comparative analysis between three promising real-time strategies, in order to evaluate their pro and cons. We focus exclusively on real-time energy management strategies and not on the underlying hardware or architectures. Thus, all strategies are applied to the same parallel HEV, in the same context. The interest of the paper is that we not only implement real-time strategies using published material, but also develop them in order to improve their performance in real-world driving conditions. The implementation issues are also described in the present paper, followed by a statistical analysis in which the three real-time strategies are evaluated over a family of stochastic driving cycles generated by Morkov process. To the best of our knowledge, this is the first time that such analysis is applied to real-time strategies in order to evaluate their real-world performance. We hope that it will encourage more researchers in the field to integrate this step into their strategy validation phase.

The paper is organized as follows: the HEV model and the principle of optimal energy management are described in Section II. The chosen strategies - three real-time strategies along with two off-line algorithms - are introduced in Section III. Section IV presents the driving cycles and the evaluation criteria which are used for comparison. The strategy implementation issues are developed in Section V. It begins with the discretization influence on the performances of the two off-line methods, which are generally considered as reference benchmark. The real-time strategies parameter determination 
phase is then presented in details, while highlighting its importance regarding the resulting fuel efficiency. Section VI discusses the simulation results. After being calibrated on one certification driving cycle, the real-time strategies are then evaluated over different road-type cycles, followed by a statistical analysis using automatically generated random cycles. Finally, the paper is concluded in Section VII.

\section{HEV MODELING AND OPTIMAL ENERGY MANAGEMENT}

The present comparison is performed in the case of a full hybrid HEV, with parallel powertrain architecture (Fig. 1). It was studied in a previous work and deemed to have a very good potential for fuel consumption reduction [14].

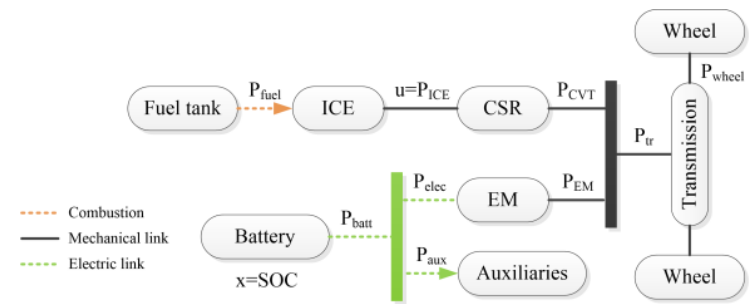

Fig. 1. Parallel hybrid electric vehicle powertrain.

\section{A. Power components modeling}

The system corresponds to a B-segment vehicle. The ICE is a 50-kW 1.0-liter 3-cylinder in-line gasoline engine modeled by a stationary brake specific fuel consumption map ( $B S F C$ in $\mathrm{g} / \mathrm{kWh}$ ) The instantaneous fuel consumption $P_{\text {fuel }}$ is then determined by (1), where $Q_{L H V}$ is the lower heating value in $\mathrm{kJ} / \mathrm{kg}$. Fig. 2 shows the resulting efficiency map.

$$
P_{\text {fuel }}=3.6 \times 10^{6} Q_{L H V} P_{I C E} B S F C
$$

A continuous speed ratio system (CSR) is used for transmission: for given ICE power $P_{I C E}$ and wheel speed $v_{\text {wheel }}$, the transmission ratio is adjusted to as to operate the ICE at its best efficiency, along the black line shown in Fig. 2.

The EM is a $50-\mathrm{kW}$ synchronous electric machine modeled by its efficiency map $\eta_{E M}=\left(\omega_{E M}, T_{E M}\right)$.

A constant power demand $P_{a u x}$ is considered for auxiliary systems.

The battery is a Li-ion one modeled by a Thevenin equivalent circuit with internal resistance $R_{\text {batt }}$ and open circuit voltage $V_{\text {oc_batt }}$.

For a given battery power $P_{b a t t}$, the battery current $I_{b a t t}$ is obtained by (2), where $E_{\text {batt }}$ is the battery energy capacity, related to the battery charge capacity $Q_{b a t t}$ according to (3). The resulting state of charge (SOC) variation is given by (4).

$$
\begin{gathered}
I_{\text {batt }}=\frac{V_{\text {oc_batt }}-\sqrt{\mathrm{V}_{\text {oc_batt }}^{2}-4 R_{\text {batt }} P_{\text {batt }}}}{2 R_{\text {batt }}} \\
E_{\text {batt }}=Q_{\text {batt }} V_{\text {oc_batt }}
\end{gathered}
$$

$$
S \dot{O} C=-\frac{I_{b a t t}}{Q_{b a t t}}
$$

In the case of charge-sustaining problems like the one considered here, the $S O C$ dependence of the battery parameters can be neglected [4].

The battery power itself depends both on the wheel power required by the driver and the ICE power which supplies part of or more than this requested power. Ignoring the efficiencies of the different transmission devices for the sake of writing simplicity, this relation is approximated by (5).

$$
P_{\text {batt }}=P_{\text {aux }}+\frac{P_{\text {wheel }}-P_{I C E}}{\eta_{E M}}
$$

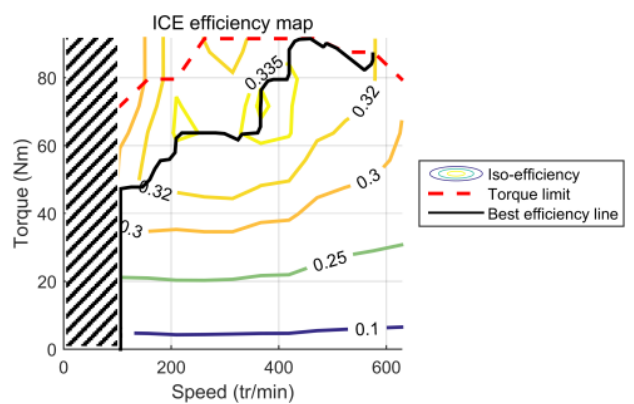

Fig. 2. ICE efficiency map. The black line corresponds to the best efficiency at a given output power; the red dotted curve indicates the maximum torque at a given speed. The minimum speed is $1010 \mathrm{r} / \mathrm{min}$.

\section{B. Optimal energy management}

The purpose of optimal power management is to search for the best power split between the ICE and the EM, in order to minimize the fuel consumption over a given driving cycle, while meeting the driver's power demand $P_{\text {wheel }}$ and maintaining the battery state of charge $S O C$.

The control and state variables are $P_{I C E}$ and $S O C$ respectively. For a less clustered writing, they will be denoted $u$ and $x$ in the rest of the paper, and $P_{w h e e l}$ will be denoted $w$.

The problem is defined by (6), (7) and (8). $J$ is the total fuel consumption over the considered time interval, while $f$ is the function which models the dynamic evolution of the system. Since the energy is provided solely by the fuel, the battery state of charge should be the same at the beginning and at the end of the trip (8). In between, the $x$ variations show how the battery is used to control the ICE working point and to recover braking energy.

$$
\begin{aligned}
& \text { Minimize } J=\int_{t_{0}}^{t_{f}} P_{f u e l}(u(t)) \cdot d t \\
& \text { subject to } \dot{x}(t)=f(u(t), w(t)) \\
& \text { and } x\left(t_{0}\right)=x\left(t_{f}\right)=x_{\text {ref }}
\end{aligned}
$$


In the case of off-line optimization, the driving cycle is fully known in advance and two mathematical approaches exist for solving the problem: Pontryagin's minimum principle (PMP) and dynamic programming (DP) [4]. PMP is very easy to implement and fast, but does not allow to easily account for constraint on the state variable. It may also fail to exactly respect the constraint on the final state of charge, as we will discuss it in Section V. On the other hand, DP is a much more cumbersome method, but has better robustness and can handle $x$ limitations when needed.

In real world, however, the driving cycle cannot be known in advance and so-called "real-time" or "on-line" energy management methods are needed. The optimal consumption and $x$-sustaining constraint cannot be guaranteed because the information required for that is not available, but one can aim at a near-optimal strategy that decreases the fuel consumption while taking the final battery state of charge $x_{f}=x\left(t_{f}\right)$ close to its reference value $x_{\text {ref }}$.

\section{REAL-TIME CONTROL METHODS}

Many real-time energy management strategies have been developed in literature. In this paper, we focus on three of them, all recently published, whose authors reported excellent performances compared to previous work. The first one, called adaptive equivalent consumption minimization strategy (A-ECMS) [3] is derived from PMP. The second, called optimal control law (OCL) [5], applies the theory of non linear optimal control theory to the considered system. Last, stochastic dynamic programming (SDP) generalizes DP in the case where the driving cycle to come can be characterized from a statistical point of view [11]. The two off-line methods, namely PMP and DP, were used to provide reference results in order to evaluate the performances of the in-line methods. The principles of the different methods are presented in this section.

A. Pontryagin's minimum principle (PMP) and adaptive equivalent consumption minimization strategy (A-ECMS)

As the A-ECMS derives from PMP, this method is first presented [17]. Let us introduce the Hamiltonian function related to (6) and (7) and defined by (9), where $p(t)$ is the co-state linked to the state equation of the system.

$$
H(p, u, x, w)=P_{f u e l}(u)+p(t) \cdot f(x, u, w)
$$

PMP states that if $u^{*}$ is the solution of problem (6) - (8), there exists a co-state $p(t)$, whose dynamics is governed by (10) and such that (11) and (12) hold.

$$
\begin{gathered}
\forall t \in\left[t_{0}, t_{f}\right] \quad \frac{d p}{d t}(t)=-\frac{\partial \mathcal{H}}{\partial x}(x, u, p, w) \\
\forall t \in\left[t_{0}, t_{f}\right] \quad \frac{\partial \mathcal{H}}{\partial u}(x, u, p, w)=0 \\
x\left(t_{0}\right)=x\left(t_{f}\right)=x_{r e f}
\end{gathered}
$$

In the present problem, the $S O C$ dependence on the battery parameters is negligible; hence the functions $f$ and $H$ have no $x$-dependence, which reported in (10) leads to a constant co-state over time: $p(t)=p$. Assuming that $H$ is a convex function, (9) - (11) can be rewritten as (13).

$$
\forall t, u^{*}(t)=\underset{u}{\arg \min }\left[P_{\text {fuel }}(u)+p . f(u, w)\right]
$$

The co-state $p$ is interpreted as the equivalent fuel cost of the battery power. $P_{\text {fuel }}$ is an increasing function of $u$, whereas $f$ is a decreasing one (for a given wheel power $w$, the more power comes from the ICE, the less from the battery). Hence the global shape and the position of the minimum are governed by the co-state $p$. Solving (13) gives the optimal command corresponding to any given value of $p$. A low value of the co-state $p$ favors the use of electric power and depletes the battery (low final battery state of charge $x_{f}$ ), whereas on the contrary a high value saves electric energy and leads to a high $x_{f}$. PMP method consists in finding an intermediate value which respects the charge sustaining constraint (12). The $x_{f}$ being an increasing function of $p$, the intermediate value is easily determined by a binary search algorithm [1].

This method is also referred to as "equivalent consumption minimization strategy" (ECMS) and was intuitively proposed before establishing its mathematical background through the PMP [18]. However, it should be noted that PMP requires a convex Hamiltonian function to guarantee global optimality [4]. This strong assumption is only partially fulfilled for the considered system, as it will be shown in Section V.

Since PMP method requires the full driving cycle knowledge, it is not suitable for in-line energy management. Many adaptive ECMS were proposed [2][3][5][7]: the idea is to estimate in real-time an equivalent cost $p$ which will allow fuel consumption reduction while insuring that the state of charge remains in acceptable boundaries. Most existing methods rely on an empirical feedback on the current $x$. The main problem of this approach is that ECMS results are extremely sensitive to the value of the equivalent factor, which leads to unstable behaviors [5].

The present paper focuses on the algorithm proposed in [3], for which interesting results are reported. The value of the equivalent cost is adjusted at regular intervals of time $T$, with a correction proportional to the difference between the current and reference $x$. A new value of $p$ is calculated for each period $[k T,(k+1) T]$ by using (14), where $K_{p}$ is the gain of the proportional controller and $x$ denotes the battery state of charge.

$$
p_{k+1}=\frac{p_{k-1}+p_{k}}{2}+K_{p} \cdot\left(x_{r e f}-x(k T)\right)
$$

The parameters of the algorithm are the refreshment period $T$, the gain $K_{p}$ and the initial guesses $p_{0}$ and $p_{1}$. Using the value of $p$ at the two previous time steps stabilizes the system behavior. It should be noted that the authors show good results, but do not say anything about the value of those parameters, nor about the procedure to determine them. 


\section{B. Optimal control law (OCL)}

ECMS are basically empirical methods. An attempt of a more rigorous approach, based on non-linear regulation and disturbance rejection, was proposed in [5]. The authors use analytical close-form of the power components in order to establish a state feedback control law which guarantees optimality and asymptotic stability.

The ICE consumption is approximated by the Willans line model, which states that at given speed $\omega_{I C E}$, the input power $P_{\text {fuel }}$ is as an affine function of the output power $P_{I C E}$ (15). Fig. 3 shows the Willians lines that correspond to the present ICE whose efficiency map has been shown in Fig. 2. The OCL method simplifies the Willans model and neglects the speed dependence of the intercept $e_{0}$ and the slope $e_{1}$.

$$
P_{\text {fuel }}\left(\omega_{I C E}, P_{I C E}\right)=e_{0}\left(\omega_{I C E}\right)+e_{1}\left(\omega_{I C E}\right) \cdot P_{I C E}
$$

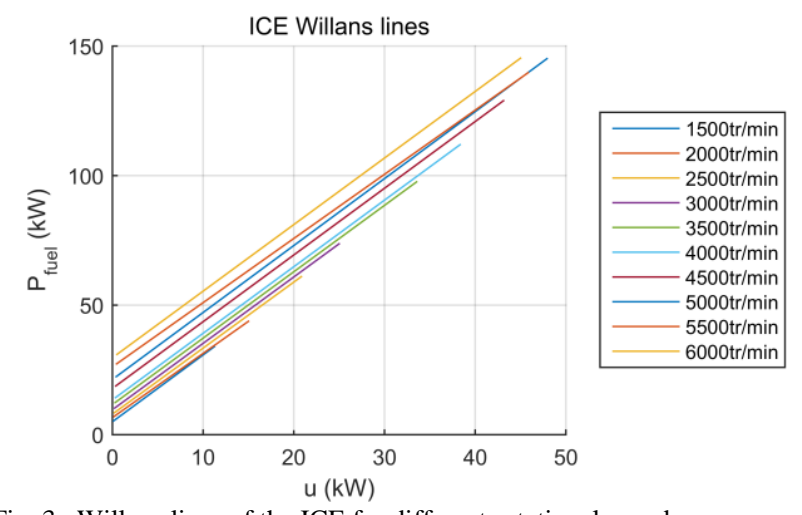

Fig. 3. Willans lines of the ICE for different rotational speeds $\omega_{I C E}$.

The electric machine and the battery are modeled by their average efficiency, denoted $\overline{\eta_{E M}}$ and $\overline{\eta_{\text {batt }}}$ respectively

Let us denote $\xi$, the difference between the reference and current SOC: $\xi(t)=x_{\text {ref }}-x(t)$. The optimal control law is given by (16), where the coefficient $p_{3}$ and $K$ are defined by (17) and (18). The only setting parameter is the constant $\mu$, which has to be determined in order to fulfill the battery charge sustaining constraint.

$$
\begin{gathered}
u^{*}=\frac{(\mu \xi)^{2}}{36\left(K \mu \xi+p_{3}\right)} \\
p_{3}=\frac{e_{1}}{Q_{L H V}} \\
K=\frac{\overline{\eta_{b a t t}}}{E_{b a t t} \cdot \overline{\eta_{E M}}}
\end{gathered}
$$

One should notice that the control variable $u^{*}$ directly depends on $\xi$. Since no minimization is needed at each step of time, the method is faster than A-ECMS for implementation on real vehicles. Furthermore, the theoretical context is clear and there is only one tuning parameter, with respect to which the method is not over-sensitive.
C. Dynamic programming $(D P)$ and stochastic dynamic programming $(S D P)$

DP is a multi-stage decision-making process which allows solving optimization problems that can be broken down into several sub-problems of the same nature [19]. It applies well to the optimization of cumulative costs in dynamic systems, such as (6) - (8).

DP requires the problem to be discretized in time and state. Let us denote respectively 0 and $N$ the indexes of the initial and final time steps, $x_{k}=\operatorname{SOC}\left(t_{k}\right), u_{k}=P_{I C E}\left(t_{k}\right)$ and $w_{k}=$ $P_{\text {wheel }}\left(t_{k}\right)$.

The discretized problem is given by (19), (20) and (21).

$$
\begin{aligned}
& \text { Minimize } J=\sum_{k=0}^{N-1} P_{f u e l}\left(u_{k}\right) \cdot \Delta t \\
& \text { subject to } x_{k+1}=x_{k}+f\left(u_{k}, w_{k}\right) \cdot \Delta t \\
& \text { and } x_{0}=x_{N}=S O C_{r e f}
\end{aligned}
$$

A so-called cost-to-go function, denoted $J_{k}(x)$, is defined at each time step $t_{k}$. It corresponds to the minimum cost that can be obtained by optimal control from a given state at time $t_{k}$ $\left(x_{k}=x\right)$ to the final state $x_{N}$. This cost is calculated backwards, starting from the final time (22) and applying the recursive process (23) where $u_{k}{ }^{*}(x)$ denotes the optimal control at time $t_{k}$ as a function of the current system state $x_{k}=x$.

$>$ Initialization: at $k=N$

$$
J_{N}(x)=100\left(x-x_{r e f}\right)^{6}
$$

$>$ Backward iterative process: from $k=N-1$ to $k=0$

$$
\begin{gathered}
J_{k}(x)=\min _{u}\left\{P_{\text {fuel }}(u)+J_{k+1}\left(x+f\left(u, w_{k}\right) \cdot \Delta t\right)\right\} \\
u_{k}^{*}(x)=\underset{u}{\operatorname{argmin}}\left\{P_{\text {fuel }}(u)\right. \\
\left.+J_{k+1}\left(x+f\left(u, w_{k}\right) \cdot \Delta t\right)\right\}
\end{gathered}
$$

$J_{N}(x)$ is a penalty function on the final state, which favors $x_{N}=x_{r e f}$. At the end of the backward process, $J_{0}(x)$ represents the minimum fuel consumption which can be obtained starting from the initial state $x_{0}=x$. The optimal control policy $u^{*}=\left\{u_{k}{ }^{*}, 0 \leq k \leq N-1\right\}$ is rebuilt by a forward process.

PMP and DP are equivalent when the Hamiltonian function is convex, and their relationship is given in [20].

SDP was developed to account for random perturbations in the system. In the present case, the uncertainty about the driving cycle can be modeled by a random process, with more or less information available about the coming itinerary. At each time $t_{k}$, the wheel power $w_{k}$ can then be described as a random variable characterized by its probability law. This uncertainty is accounted for by an average cost (24) where 
$E_{w_{k}}[\cdot]$ represents the mathematical expectation with respect to $w_{k}$ probability law

$$
J=E_{w_{k}}\left[\sum_{k=0}^{N-1} P_{\text {fuel }}\left(u_{k}\right) \cdot \Delta t\right]
$$

The cost-to-go function $J_{k}\left(x_{k}\right)$ is defined the same way by (25) and corresponds to the minimum average cost that can be obtained by optimal control from the state $x_{k}$ at time $t_{k}$ to the final state $x_{N}$.

$$
\begin{aligned}
J_{k}(x)=\min _{u}\left\{E_{w_{k}}\right. & {\left[P_{\text {fuel }}(u)\right.} \\
& \left.\left.+J_{k+1}\left(x+f\left(u, w_{k}\right) \cdot \Delta t\right)\right]\right\}
\end{aligned}
$$

The iterative process described by formula (22) and (23) remains the same, except that an additional loop is needed to calculate the cost as a mathematical expectation.

At the end of the process, $J_{0}(x)$ represents the average optimal fuel consumption from the initial to the final state $x_{0}=x$. The forward process is applied to the in-line driving cycle, that is to say a given realization of the random process. It builds the optimal control policy $u^{*}=\left\{u_{k}{ }^{*}, 0 \leq k \leq N-1\right\}$ for the corresponding realization of the random driving cycle. The actual cost may not be the lowest one for the considered cycle, but it is in an average sense.

From a practical point of view, accounting for the random nature of the driving cycle adds complexity and computational load, but it should be underlined that it affects only the backward part of the algorithm, when all possible realizations of the random process are evaluated. This part is done only once and off-line. During the in-line process, only the forward part of the algorithm is applied to the actual driving cycle, and it basically consists in searching the command in a look-up table.

SDP is a well established method, but the quality of the results relies on the quality of the random process model. In the considered problem, a good statistical representation of the driving cycles is needed, and this is a challenging problem. In a previous work [21], the driving cycle was modeled by a random speed characterized by a normal distribution $N(\mu, \sigma)$, where the parameters $\mu$ and $\sigma$ were inferred from driving cycle data by the maximum-likelihood estimation, as proposed in [15]. This approach gave disappointing fuel saving performances, which can be explained by the fact that the vehicle instantaneous speed is not enough to predict realistic driving cycles. In the present paper, a more sophisticated approach, first proposed in [8], is applied: the power demand at a given time is modeled by a Markov process and assumed to depend on both the power demand and the vehicle velocity at the previous time. This model will be detailed in Section V.

\section{COMPARISON APPROACH AND CONSUMPTION CRITERION}

The different strategies have been implemented, tested and compared for the considered system and a large number of driving cycles. PMP and DP provide reference results, in terms of lowest consumption reachable for a given driving cycle and assess the performances of the three real-time strategies.

\section{A. Driving cycles}

A key point for a significant real-time strategies evaluation is to perform tests with data which are different from the one used for identification. In order to be convincing, conclusions must rely on statistical analysis involving numerous driving cycles, but most published works do not report this kind of results. The present study tries to fulfill this lack and give a broad overview of the different strategies behaviors by testing them for a wide range of driving cycles, corresponding to different driving conditions.

The first cycle to be considered is a certification one. As the current official European driving cycle (NEDC) is known to poorly represent real world driving behavior, the forthcoming Worldwide harmonized Light vehicles Test Cycle (WLTC) cycle is preferred (Fig. 4) [22]. This cycle cumulates the three types of driving environment: city, road and highway.

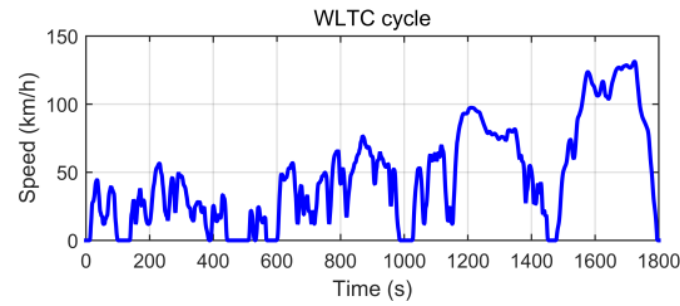

Fig. 4. WLTC driving cycle ( $23 \mathrm{~km}$ long, $47 \mathrm{~km} / \mathrm{h}$ average speed)

Then, a series of ten INRETS cycles representative of user behavior [23] is used for robustness analysis. Their characteristics are reported in TABLE I: each of these cycles corresponds to a specific driving context, enabling to test the strategies behavior and performances in all possible conditions.

TABLE I

INRETS CYCLE CHARACTERISTICS

\begin{tabular}{c|ccccc|ccc|ccc}
\hline \hline Type & \multicolumn{5}{c|}{ Urban } & \multicolumn{3}{c|}{ Road } & \multicolumn{2}{c}{ Highway } \\
\hline Cycle & UL1 & UL2 & UF1 & UF2 & UF3 & R1 & R2 & R3 & A1 & A2 \\
\hline $\begin{array}{c}\text { Average } \\
\text { speed } \\
(\mathrm{km} / \mathrm{h})\end{array}$ & 4 & 7 & 10 & 19 & 24 & 32 & 41 & 57 & 74 & 95 \\
\hline $\begin{array}{c}\text { Travel } \\
\text { distance } \\
(\mathrm{km})\end{array}$ & 1 & 2 & 2 & 6 & 7 & 8 & 9 & 16 & 15 & 26 \\
\hline \hline
\end{tabular}

The last step is to evaluate the strategies for a large number of real-world representative missions, as they could be forecasted using in-line traffic information [7][24][25]. For this purpose, random driving cycles were generated using a Markov-chain model fed by the statistical characteristics of the WLTC or INRETS cycles.

For each driving cycle, the wheel power is calculated using the dynamic equation of the vehicle: 


$$
P_{w h e e l}=\left(\frac{1}{2} \rho_{a i r} A C_{d} v_{w h e e l}^{2}+\mu_{r} M g+M a\right) v_{w h e e l}
$$

where $\rho_{\text {air }}$ is density of air; $A$ is the reference area; $C_{d}$ is the drag coefficient; $\mu_{r}$ is the rolling resistance coefficient; $M$ is the vehicle mass; $g$ is the gravitational acceleration; $a$ is the vehicle acceleration.

\section{B. Comparison criteria}

The main comparison criterion is the fuel consumption over the driving cycle:

$$
J^{*}=\int_{t_{0}}^{t_{f}} P_{\text {fuel }}\left(u^{*}(t)\right) \cdot d t
$$

However, the final battery state of charge $x_{f}=x\left(t_{f}\right)$ must also be accounted for, as an indicator of the charge sustaining constraint respect. As mentioned in Section II, the $x$-sustaining constraint cannot be guaranteed in real-time optimization because the future driving information is not available in advance. As a result, two consumption values calculated by (27) cannot be compared if they correspond to different $x_{f}$. Hence, comparison between the strategies is done using a corrected consumption which includes the electric energy stored in the battery. This corrected consumption is defined by (28), where $\eta_{s y s}$ is the overall system efficiency calculated by (29).

$$
\begin{gathered}
J_{\text {cor }}^{*}=J^{*}+\left(x_{\text {ref }}-x_{f}\right) \cdot \frac{E_{\text {batt }}}{\eta_{\text {sys }}} \\
\eta_{\text {sys }}(\%)=\frac{\int_{t_{0}}^{t_{f}}\left(P_{\text {wheel }}+P_{\text {aux }}\right) d t}{J^{*}} \times 100 \%
\end{gathered}
$$

For the WLTC cycle (23 km long), a 5\% $x$-difference corresponds to a 0.1 liter $/ 100 \mathrm{~km}$ consumption if the overall efficiency is $20 \%$ and to 0.2 liter $/ 100 \mathrm{~km}$ if it is $10 \%$.

The fuel saving is calculated with respect to the consumption of a conventional vehicle (CV) driving the same cycle as follows:

$$
\text { Fuel saving }(\%)=\frac{J_{H E V}-J_{C V}}{J_{C V}} \times 100 \%
$$

A positive fuel saving value means that the HEV burns more fuel than the $\mathrm{CV}$ over the same driving mission while a negative value means the HEV actually economizes.

\section{IMPLEMENTATION ISSUES}

The current section addresses practical issues for a proper numerical implementation of the different optimization algorithms. The two off-line algorithms - PMP and DP - are generally considered as benchmarks to evaluate real-time strategies. As references, their fuel consumption must be carefully calculated and one must obtain the same results for the same system model and driving cycles. However, the numerical implementation of these algorithms brings on discretization effects whose influence will be analyzed

Then the problem of setting parameters determination is discussed. It should be noted that authors usually do not give much information about this procedure, although it is an important point for a good implementation of each strategy. In the present paper, the parameter setting influence on strategy performance will be discussed in details

\section{A. PMP numerical implementation}

As mentioned in Section III, PMP method determines the optimal co-state value $p$ by a binary search algorithm using the charge sustaining constraint. This algorithm applies well because the final state $x_{f}$ is an increasing function of the equivalent cost $p$, as shown by Fig. 5, which shows the values of $p$ tested during the iterative process and the progressive reduction of the research interval. Yet, magnifying the curve reveals local discontinuities and a staircase shape. A local jump exists in the interval $[141.5107,141.5108]$ and forbids to exactly fulfill the constraint $x_{f}=x_{r e f}=40 \%$.
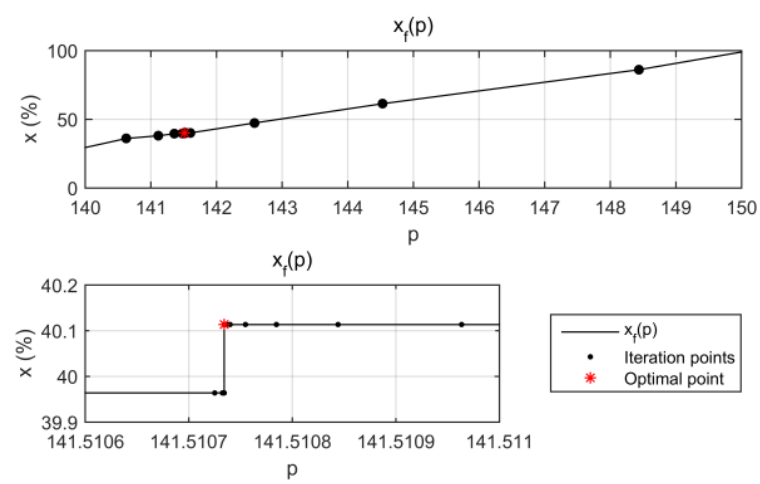

Fig. 5. Final battery state $x_{f}$ versus equivalent cost $p$ and close-up: points calculated during the binary search to reach $x_{f}=x_{r e f}=40 \%$.

Such jumps exist all over the $x_{f}(p)$ curve and can easily be explained. For each co-state value $p$, the optimal control sequence requires to solve (13) and minimize the Hamiltonian function at each time step. It turns out that this function is not convex everywhere and sometimes has two local minima, as shown in Fig. 6.

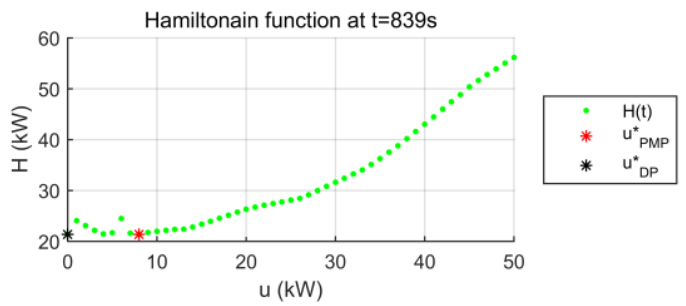

Fig. 6. Hamiltonian function at $t=839 \mathrm{~s}$ of the WLTC cycle, for $p=141.5$. Two local minima exist, with very close values of $\mathrm{H}$. The red $u_{P M P}^{*}$ point is the instantaneous control taken by PMP method and the black one is chosen by DP.

The algorithm returns the smallest one, but in this situation, an infinitely small change of $p$ produces a switch from one minimum to the other and a discontinuity of the command, 
which in turn impacts the final state $x_{f}$. The non convexity is especially marked at the origin, as the ICE is turned off for $u=0$.This fact was also mentioned and analyzed in [26]. The jumps are particularly pronounced in the case of driving cycles with unnatural flat zones, such as NEDC.

PMP algorithm can be very efficiently written by using the matrix formalism and functions available in Matlab® or any environment of this type. Time consuming loops can be avoided, excepted the one needed for the binary search. This numerical approach requires the use of discrete command values $\left[u_{i}\right]_{0 \leq u_{i} \leq 50 \mathrm{~kW}}$ among which the optimal one is chosen.

Fig. 7 and Fig. 8 show the influence of the command discretization $d u$ on the optimal equivalent $\operatorname{cost} p$, the fuel consumption and the final state $x_{f}$, in the case of the WLTC driving cycle. The equivalent cost decreases when the discretization is refined, which favors the use of electric power and decreases the fuel consumption. A 0.07 liter $100 \mathrm{~km}$ difference between $d u=1 \mathrm{~kW}$ and $d u=10 \mathrm{~W}$ is observed, whereas the computation time increases from $0.1 s$ to $5 s$. The final state has an erratic behavior, which is not surprising. In the rest of the study, most calculations will be done with $d u=1 \mathrm{~kW}$, as it gives a good compromise between precision and computation time, but one must keep in mind that the optimal consumption is slightly overvalued.

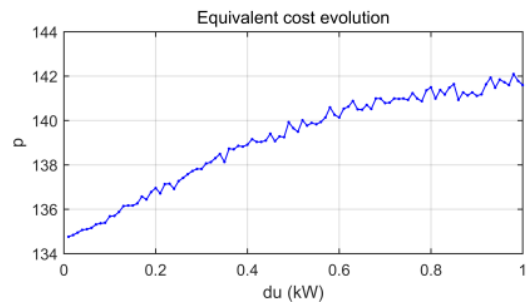

Fig. 7. Influence of the command discretization du on the equivalent cost.
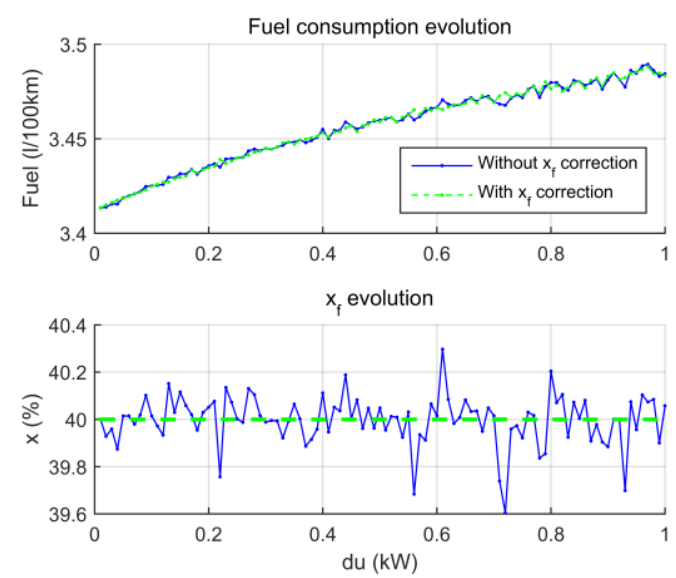

Fig. 8. Influence of the command discretization $d u$ on the fuel consumption and final state $x_{f}$ (WLTC cycle).

\section{B. DP algorithm implementation}

DP is a more complex method, but is a global optimization method which better explores the solution space and always meets the SOC-sustaining requirement. However, it also suffers from discretization problems: a fine discretization achieves a better performance but requires more computation time. This is the main reason why DP is often abandoned in favor of PMP [2]. However, DP computation load is often exaggerated in the literature, when people report that its computation time exponentially increases with the number of states [10]. In fact, the number of operations is proportional to $m n^{2} N$, where $m$ is the number of controls, $n$ is the number of states and $N$ is the number of time steps [19]. The computation time can be optimized by proper coding using matrix formalism and parallelization in Matlab®. In our case, running DP for the WLTC cycle with $d u=1 \mathrm{~kW}$ and $d x=0.1 \%$ takes only $2 \mathrm{~s}$.

At time $\mathrm{k}$, a given command $u_{k}$ produces a certain change of state according to the evolution law of the system (20). Interpolation errors can be limited by using consistent state and command discretization steps $\mathrm{dx}$ and $\mathrm{du}$, that is by choosing $d x \cong f\left(d u, w_{k}\right) \Delta t$. The evolution law is not linear, so that this relationship provides only consistent order of magnitudes of both discretization steps. For the considered system, it is found that a good correspondence is $d x \cong 1 / 36 d u$, where $d x$ and $d u$ are expressed in \% and $k W$ respectively.

Fig. 9 and Fig. 10 show the influence of the discretization steps on the fuel consumption: for usual step sizes, both $d u$ and $d x$ are important (Fig. 9), but for refined discretization, $d x$ dominates (Fig. 10). We observe an $0.13 \mathrm{l} / 100 \mathrm{~km}$ consumption difference between $d u=1 \mathrm{~kW}, d x=0.1 \%$ and $d u=10 \mathrm{~W}, d x=3.10^{-4} \%$ over the WLTC cycle. The figures also show a good $x$-sustaining respect, and thus there is no need for $x_{f}$ correction.

To sum up, PMP and DP have similar minimization performance. DP is able to meet the SOC-sustaining constraint perfectly, but it requires much more computational time. Besides, this method remains interesting if one needs to account for SOC limitation [14]. Considering the strategy discretization coherence and the resulting SDP computational time increase, the discretization sizes $d u=1 \mathrm{~kW}$ and $d x=$ $0.1 \%$ are taken for strategy comparison in the rest of the study.
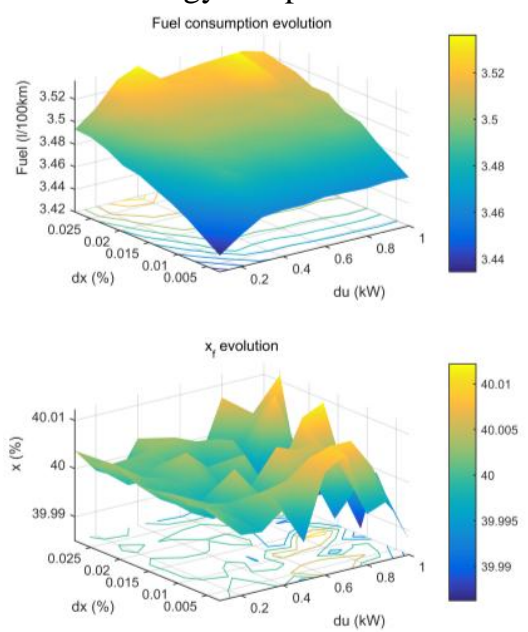

Fig. 9. Fuel consumption and final state with the command and state discretizations $d u=[0.1,1]$ and $d x=1 / 36 . d u$ 

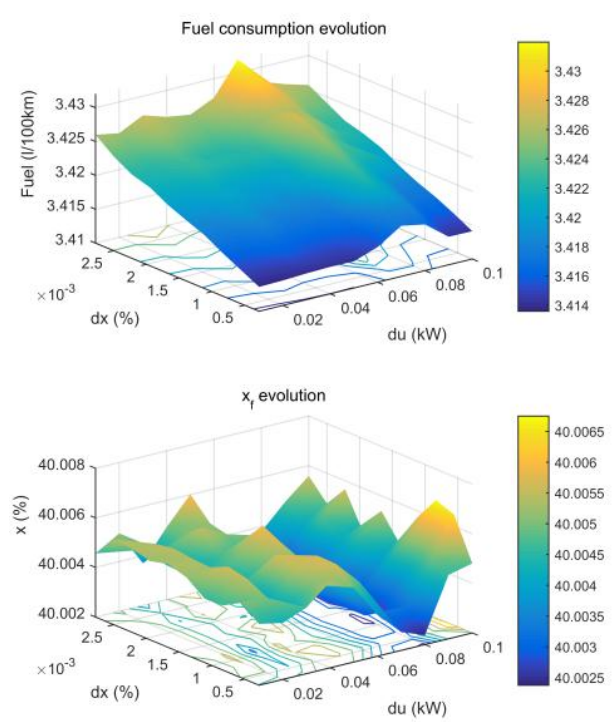

Fig. 10. Fuel consumption and final state with the command and state discretizations $d u=[0.01,0.1]$ and $d x=1 / 36 . d u$

\section{A-ECMS parameters determination}

The performances of ECMS-type methods are known to be very sensitive to the value of the equivalent cost [5], making it always difficult to determine their parameters. The parameters should be determined once, using a given cycle representative of the different situations to come. In the present section, the parameters are calculated using the WLTC certification cycle.

In the case of the A-ECMS method, the setting parameters are the period $T$, the gain $K_{p}$ and the initial guesses $p_{0}$ and $p_{1}$ and a procedure must be given in order to determine them in an efficient way. For a given driving cycle, the A-ECMS consumption $J^{*}$ and the resulting final battery state of charge $x_{f}$ depend on the setting parameters, and the procedure must allow to determine the set of parameters which insures both consumption reduction and $x_{f}$ constraint respect.

To do this, we define a cost function $J_{G A}$ (31) which aggregates both objectives. The setting parameters are calculated by minimizing this function using a genetic algorithm (GA) (Matlab "ga" function).

$$
\begin{gathered}
J_{G A}\left(T, K_{p}, p_{0}, p_{1}\right)=J^{*}+\text { penalty }\left(x_{f}\right) \\
\operatorname{penalty}\left(x_{f}\right)=100\left(x_{f}-x_{\text {ref }}\right)^{6}
\end{gathered}
$$

The number of parameters was reduced by choosing $p_{0}=p_{1}$. Inequality constraints are set to define the searching space: $1 \leq p_{0} \leq 200,1 \leq T \leq 60 s$ and $1 \leq K_{p} \leq 10$.

GA setting parameters influence itself was carefully examined. Fig. 11 displays the evolution of the criteria $J_{G A}$ as a function of the generation number, for population sizes ranging between 20 and 300. It shows that no gain is obtained after 25 generations. Obviously, many local minima exist and the algorithm is rapidly trapped, even with the largest population size. Very close fuel consumptions (differences less than $0.1 \mathrm{l} / 100 \mathrm{~km}$ ) are obtained with quite different set of parameters. Fig. 11 illustrates the dispersion of the resulting setting parameters in the $\left(T, K_{p}, p_{0}\right)$ space.

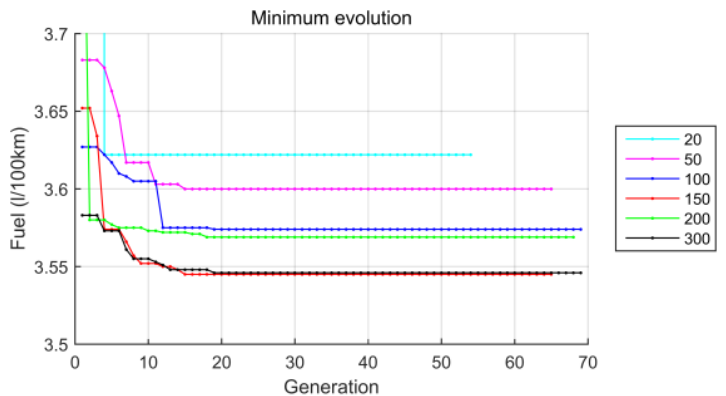

Fig. 11. Equivalent consumption $J_{G A}$ versus number of generations, for population sizes ranging between 20 and 300 .

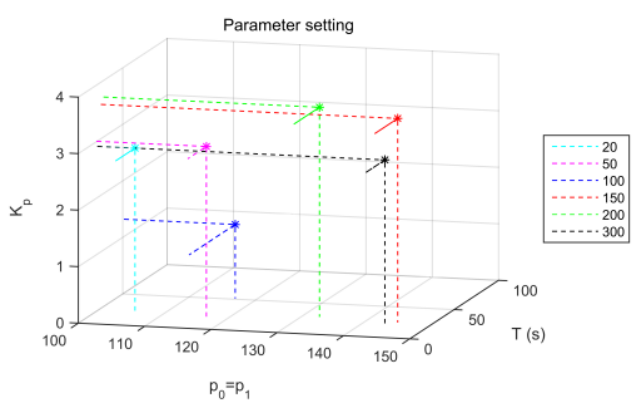

Fig. 12. A-ECMS parameters found after 100 generations, for population sizes ranging between 20 and 300 .

After this preliminary study, GA was run with a population size of 100 individuals and 25 generations. Fig. 13 shows the evolution of the criteria $J_{G A}$ as a function of the generation number, for five different initial populations. It confirms the existence of local minima and the dispersion of the corresponding parameters.

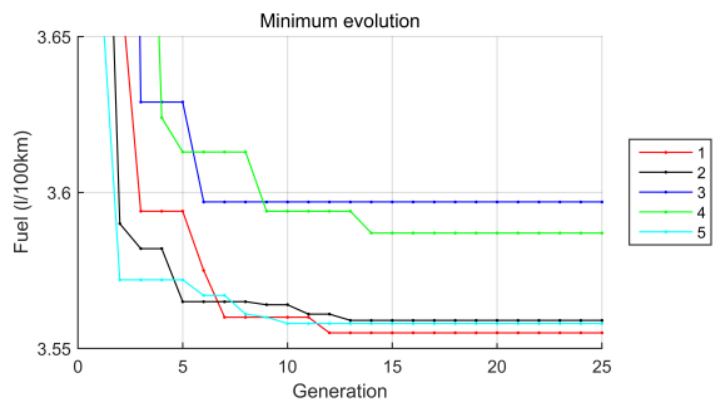

Fig. 13. Equivalent consumption $J_{G A}$ versus generation number, for five different initial populations.

The A-ECMS method was applied to the WLTC cycle with the 5 sets of parameters found by the genetic algorithm and the results are reported in TABLE II. The parameters set \#2 is chosen for the strategy comparison conducted in Section VI because it reaches the lowest consumption while satisfying 
exactly the battery charge sustaining constraint.

TABLE II

A-ECMS RESULTS FOR FIVE DIFFERENT PARAMETER SETS

\begin{tabular}{cccccc}
\hline \hline Set \# & $T$ & $K_{p}$ & $p_{0}, p_{1}$ & $\begin{array}{c}J^{*} \\
(1 / 100 \mathrm{~km})\end{array}$ & $x_{f}$ \\
\hline 1 & 38 & 4.7 & 134.1 & 3.55 & 39.8 \\
2 & 16 & 2.1 & 139.0 & 3.56 & 40.0 \\
3 & 47 & 1.5 & 167.9 & 3.58 & 40.3 \\
4 & 37 & 4.3 & 74.3 & 3.59 & 40.0 \\
5 & 45 & 1.1 & 120.1 & 3.56 & 39.9 \\
\hline \hline
\end{tabular}

\section{OCL parameters determination}

The OCL method has three parameters denoted $K, p_{3}$ and $\mu$, which are easy to determine. $K$ and $p_{3}$ are calculated using physical parameters of the system (cf. Section III), whereas $\mu$ is a constant to calibrate in order to respect the battery charge sustaining constraint.

Fig. 14 shows that the final state $x_{f}$ is a monotonic increasing function of $\mu$, enabling to easily find the optimal value by binary search. For the WLTC driving cycle, $\mu=$ $63.6 \mathrm{~kg}$.

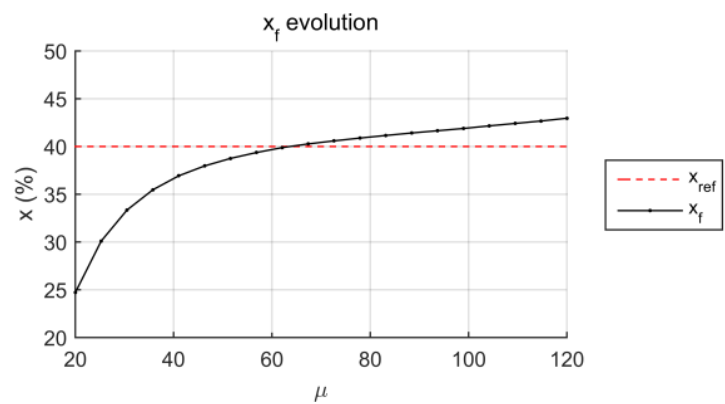

Fig. 14. Final state of charge versus parameter $\mu$, case of WLTC official cycle.

\section{E. SDP implemantation}

As discussed in Section III, SDP is a well established method, and the main problem in order to implement it is to have a good statistical model of the driving cycles [25]. We have used the stationary Markov chain model proposed in [11]. The power demand $P_{\text {wheel }}^{k+1}$ at a given time $k+1$ is assumed to depend on both the power demand $P_{w h e e l}^{k}$ and the speed $v_{w h e e l}^{k}$ at the time before $k$. The velocity $v_{w h e e l}^{k+1}$ is then determined by applying the vehicle dynamic equation.

The transition probabilities are determined as follows. The observed data of a given cycle $\left[P_{w h e e l}^{k}\right]_{k=0, N}$ and $\left[V_{w h e e l}^{k}\right]_{k=0, N}$ are mapped into a finite number of classes $n_{P}$ and $n_{V}$, corresponding to power and velocity intervals characterized by their central values $\left[P_{i}\right]_{i=1, n_{P}}$ and $\left[V_{j}\right]_{j=1, n_{V}}$. The probability to have $P_{w h e e l}^{k+1}=P_{l}$, knowing that $P_{w h e e l}^{k}=P_{i}$ and $V_{w h e e l}^{k}=$ $V_{j}$ is determined by the maximum-likelihood estimator (33),

$$
\begin{aligned}
& \mathrm{P}\left(P_{\text {wheel }}^{k+1}=P_{l} \mid V_{\text {wheel }}^{k}=P_{i}, P_{\text {wheel }}^{k}=P_{j}\right) \\
= & p_{i j, l}=\frac{m_{i j, l}}{m_{i j}}
\end{aligned}
$$

where $m_{i j, l}$ is the number of times a transition has occurred from $P_{i}$ to $P_{l}$ at vehicle velocity $V_{j}$ and $m_{i j}$ is the number of times $P_{i}$ has occurred at vehicle velocity $V_{j}$.

Fig. 15 shows an example of Markov chain transition probability map at a given speed, using the WLTC cycle as data base. The discretization sizes are $d v_{\text {wheel }}=1 \mathrm{~m} / \mathrm{s}$ and $d P_{\text {wheel }}=1 \mathrm{~kW}$. It should be noted that an adequate probability distribution of the vehicle's driving speed requires a large amount of data not always available. This can be seen as a drawback or on the contrary as a way to include more information about the current trip of the vehicle.

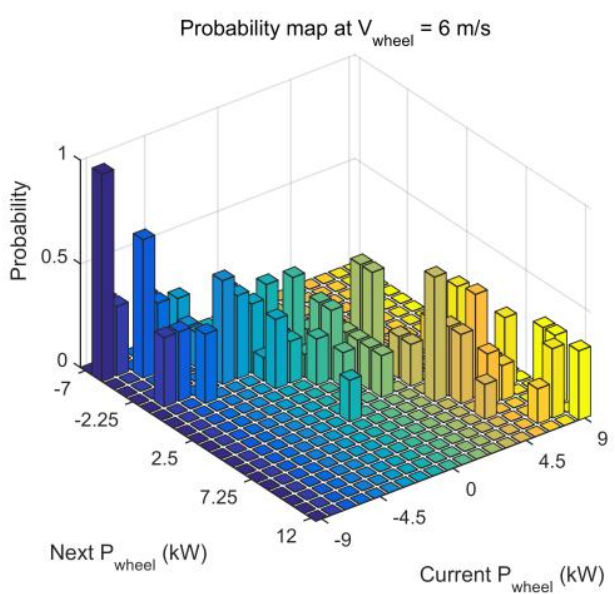

Fig. 15. Probability map at $v_{\text {wheel }}=6 \mathrm{~m} / \mathrm{s}$ for the WLTC cycle.

The discretization sizes $d v_{\text {wheel }}=1 \mathrm{~m} / \mathrm{s}, d P_{\text {wheel }}=1 \mathrm{~kW}$, $d u=1 \mathrm{~kW}$ and $d x=0.1 \%$ were chosen for strategy comparison.

It should be noticed that stochastic dynamic programming requires a large computational load. For the WLTC cycle, it takes $12 \mathrm{~h}$ with the parameter setting indicated above and one can notice that it is not a fine discretization at all. However, most of the computation time is taken by the backward part of the algorithm and the computation of the cost-to-go function $J_{k}$, which is done off-line only for calibration cycles. The in-line part consists in applying a look-up table command and is as fast as classical dynamic programming.

\section{SimUlation RESULTS AND DISCUSSION}

This section presents the results obtained with the three real-time strategies under study, in various test conditions. The setting parameters were determined as explained in the previous section, using data of the reference WLTC cycle. PMP and DP results serve as target results. In Sub-section VI.A, the strategies behaviors are examined for the same WLTC cycle, in order to establish the potential of each one. In Sub-section VI.B, their robustness is tested by applying them to a series of ten driving cycles representative of various context, with 
unchanged setting parameters. Finally, Sub-section VI.C presents a statistical analysis conducted over a large number of random driving cycles, in order to evaluate the strategies performances for real-world driving conditions with parameters optimized for the different driving conditions.

\section{A. Results for the WLTC cycle}

TABLE III reports the consumptions and the final battery state of charge obtained for the WLTC cycle, which is also used for the optimal adjustment of the strategies parameters. For a better analysis of the results, Fig. 16 and Fig. 17 respectively show the different control policies and battery state of charge trajectories

TABLE III SIMULATION RESULTS FOR THE WLTC CYCLE

\begin{tabular}{|c|c|c|c|c|}
\hline Strategy & Parameters & $\begin{array}{l}\text { Fuel consumption } \\
\qquad(1 / 100 \mathrm{~km})\end{array}$ & $\begin{array}{l}\text { Fuel saving } \\
\qquad(\%)\end{array}$ & $\begin{array}{l}x_{f} \\
(\%)\end{array}$ \\
\hline PMP & $d u=1 k W$ & 3.48 & -18.7 & 40.0 \\
\hline DP & $\begin{array}{l}d u=1 k W \\
d x=0.1 \%\end{array}$ & 3.53 & -17.5 & 40.0 \\
\hline A-ECMS & $\begin{array}{c}d u=1 \mathrm{~kW} \\
p_{0}=p_{1}=139.0 \\
T=16 s ; K_{p}=2.1\end{array}$ & 3.56 & -16.8 & 40.0 \\
\hline OCL & $\mu=63.6 \mathrm{~kg}$ & 4.08 & -4.7 & 40.0 \\
\hline SDP & $\begin{array}{c}d u=1 \mathrm{~kW} \\
d x=0.1 \% \\
d v_{\text {wheel }}=1 \mathrm{~m} / \mathrm{s} \\
d P_{\text {wheel }}=1 \mathrm{~kW}\end{array}$ & 3.69 & -13.8 & 43.8 \\
\hline $\mathrm{CV}$ & - & 4.28 & - & - \\
\hline
\end{tabular}
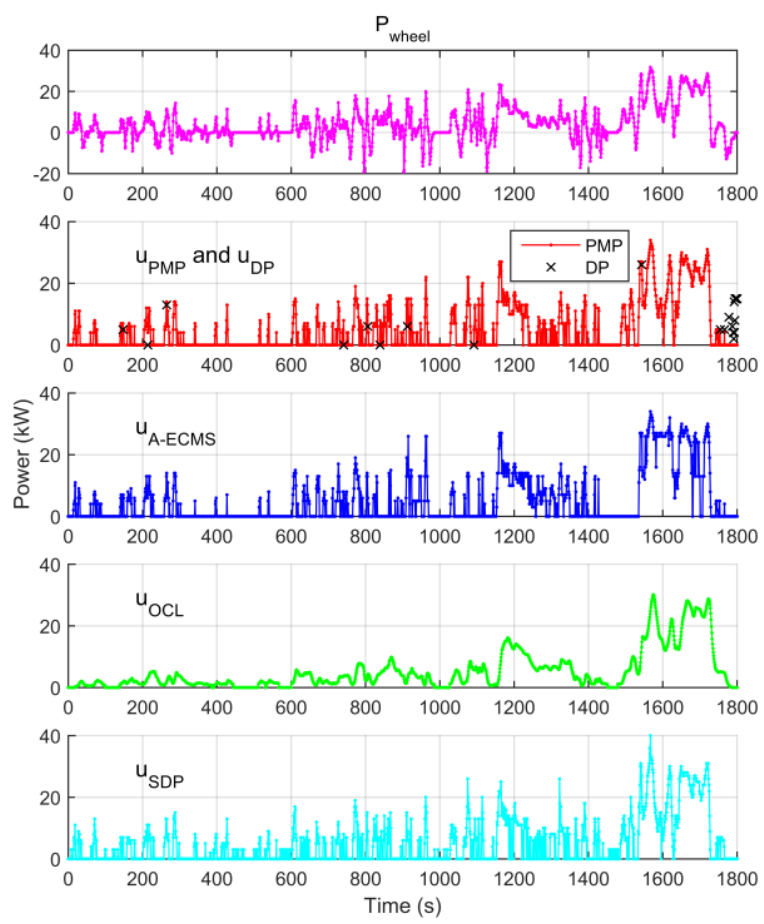

Fig. 16. WLTC power cycle and optimal control policy calculated by the different energy management strategies for this cycle.

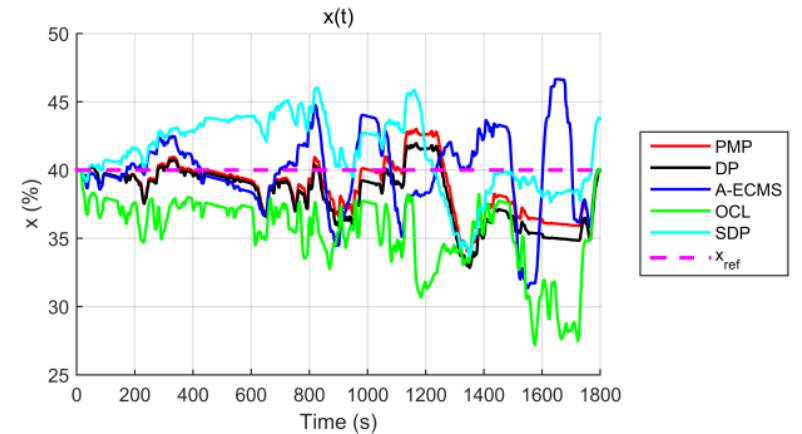

Fig. 17. Battery state of charge trajectories for the WLTC cycle.

As expected, PMP and DP give the lowest consumptions and have very similar behaviors. Yet, they differ in some isolated points and in the very last part of the WLTC cycle. Fig. 18 shows the Hamiltonian function at $t=1766 \mathrm{~s}$, one of the points where the PMP and DP commands are slightly different. We think that the initial penalty function (22) needed to initialize the cost-to-go function $J_{N}$ produces some numerical errors which lead to the observed discrepancies in the last steps of the cycle. Fig. 6 (Section V) illustrates another example of discrepancy, when the non-convexity of the Hamiltonian functions at $u=0$ produces two local minima which may lead to different commands. In the end, DP results in a slightly higher consumption, with a difference within the range of discretization error shown in Section V.

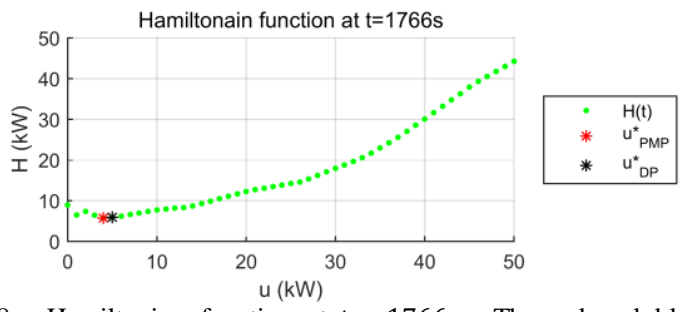

Fig. 18. Hamiltonian function at $t=1766 \mathrm{~s}$. The red and black points correspond to the ICE command calculated by PMP and DP respectively.

The SOC trajectories shown in Fig. 17 reflect well the different behaviors of the three real-time optimization strategies. The respect of the final state of charge for A-ECMS and OCL is the consequence of the fact that this constraint was used to determine the setting parameters of these strategies.

As mentioned in Section III, the A-ECMS tries to adjust the equivalent fuel-cost of electrical power around its optimal value using a state of charge feedback. This optimal equivalent cost depends on the driving cycle and can be obtained off-line using the PMP. Fig. 19 shows how the instantaneous equivalent cost calculated by A-ECMS oscillates around the optimal value calculated by PMP. These oscillations can be correlated to the ones observed on the SOC trajectory around the reference value. One notices that the consumption calculated by A-ECMS is very close to the optimal one despite different trajectories. This indicates that the optimum is not a sharp one. 


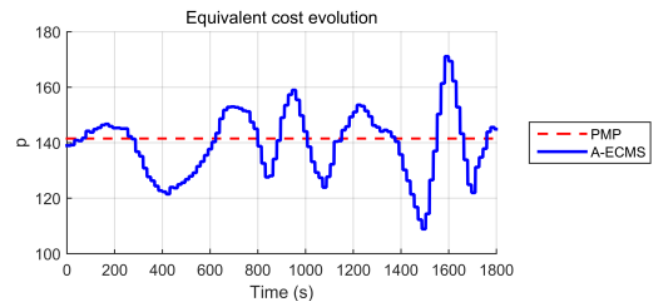

Fig. 19. Oscillation of the A-ECMS equivalent cost around the optimal value calculated by PMP.

Fig. 17 shows that OCL method favors battery discharge more than the other strategies. One also notices that this strategy often operates the ICE at a rather low power (Fig. 16), where the efficiency is not good (Fig. 2). The mechanism which consists in using the battery storage to operate the ICE at a power larger than the requested one and take advantage of a best efficiency does not seem to be activated. This is reflected by the high consumption of this strategy.

SDP performance lies between A-ECMS and OCL. The method requires a fair amount of ICE power from time to time during lower propulsion power phases to operate the ICE at a good efficiency point and maintain the battery state.

\section{B. Results in the case of INRETS cycles}

In the previous section, the methods have been tested on the same cycle that the one used to determine the setting parameters. Hence, the results are the best ones that each method can achieve. In order to establish their real-time performances, the strategies are now tested for a series of ten INRETS cycles representative of different environments, with the same setting parameters as before. Fig. 20 reports the fuel consumption saving reached by the different methods, calculated with respect to a conventional vehicle. The saving is calculated using the corrected consumption defined in (28), which accounts for the final battery state of charge, also plotted. PMP and DP methods are used to provide reference results.
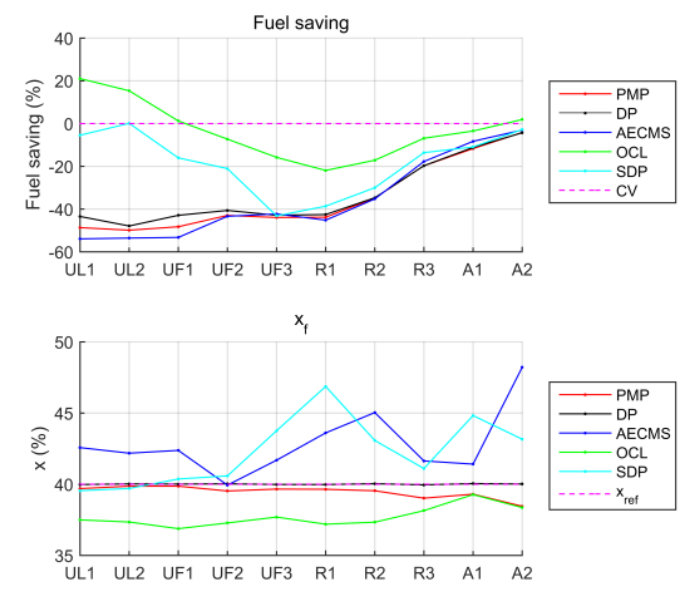

Fig. 20. Real-time strategy results for INRETS cycles, compared to off-line strategies'. Top: the fuel saving is calculated using the corrected consumption defined in (28)- Bottom: the final state $x_{f}$ indicates the capability of each method to respect the final state constraint.
The A-ECMS results show a good fuel economy using the parameters calculated for the WLTC cycle which is a combination of all three driving types. However, we have seen in Section $\mathrm{V}$ that finding the right set of parameters is not obvious because several solutions appeared during the process. For this reason, we have also tested the A-ECMS method with the 5 parameter sets reported in TABLE II. The results are presented in Fig. 21 and show significant dispersion of the consumption saving as well as the final battery state of charge. This sensitivity to the parameters is a weak point of the method, although it gives overall good results.

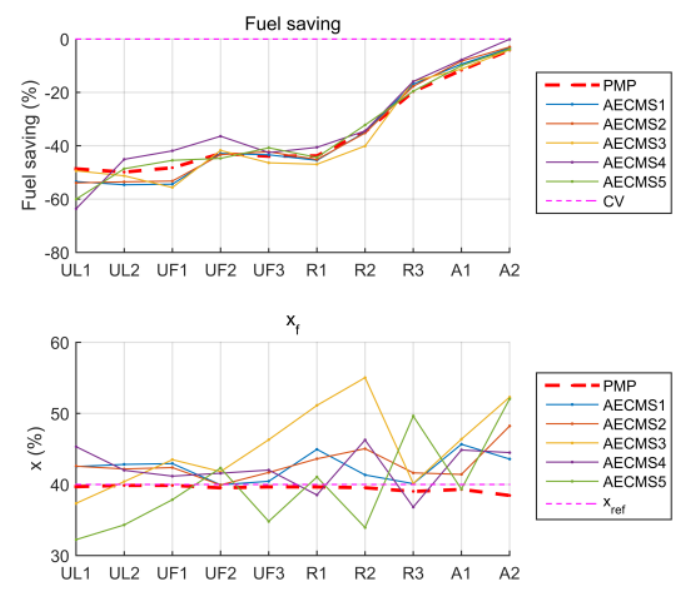

Fig. 21. A-ECMS results for INRETS cycles, with 5 different sets of parameters $\left(T, K_{p}, p_{0}\right)$. The fuel saving is calculated using the corrected consumption defined in (28).

The OCL method was developed to obtain an easier calibration than A-ECMS. According to the authors [5], it gives a solution close to the optimal one and is stable enough to work for any driving cycle with the single parameter $\mu$. The reduction of the number of parameters indeed eliminates the setting difficulty. However, the method does not allow to reduce efficiently the fuel consumption. We have tested different values of the single parameter $\mu$, but Fig. 22 shows that whatever value $\mu$ takes, OCL fails to improve the fuel consumption and insure charge sustaining at the same time: $\mu=100 \mathrm{~kg}$ gives the best charge sustaining respect, but the worst fuel consumption saving.

The SDP, a proven method in other area, uses statistics to model the driver's future power demand and calculate an average optimal solution. Our results show average performances between A-ECMS and OCL. The SDP performances rely on an adequate probability distribution of the vehicle's driving cycle. In previous Subsection VI.A, the SDP method shows a performance close to A-ECMS, but it seems that the statistical model based on the WLTC cycle is not adequate for INRETS cycles. 


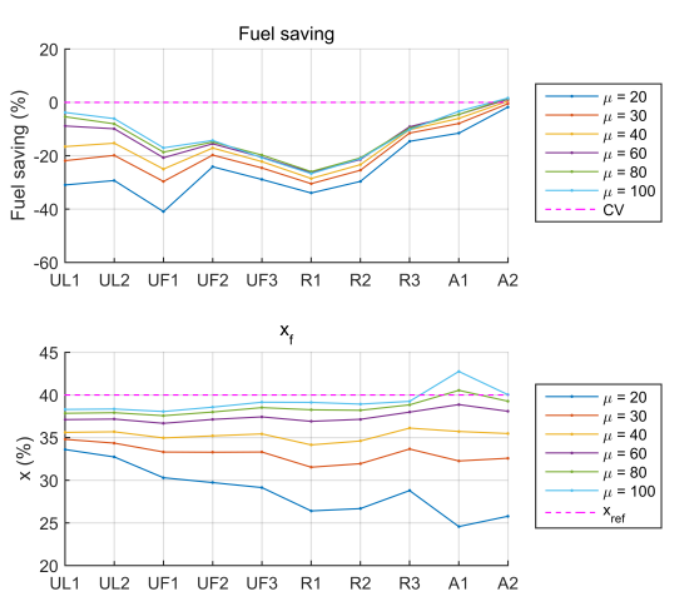

Fig. 22. OCL results for INRETS cycles, for six different values of the setting parameter $\mu$. The fuel saving does not include any $x_{f}$ correction

\section{Statistical analysis with random cycles}

In this section, the Markov chain model built for SDP method is used as a random cycle generator, with given statistical properties. A sensitivity analysis is performed on the basis of 1000 different cycles generated for the eight INRETS cycles denoted UF1 to A2 in TABLE I. The cycles UF1 and UF2 are left out because of their extremely low average speed and short travel distance. This represents a database with 8000 driving cycles. Each cycle is $1000 s$ long. This length was chosen after checking that it is long enough to insure a correct statistical representation of each type of context.

The parameters of the three tested strategies were optimized for each INRETS cycle, in order to establish to what extend the performances can be improved by adjusting the parameters to the current driving context. The parameters sets are given in TABLE IV.

TABLE IV

REAL-TIME STRATEGY PARAMETER SETTINGS

\begin{tabular}{cccc}
\hline \hline Cycle & A-ECMS & OCL & SDP \\
\hline UF1 & $\begin{array}{c}p_{0}=p_{1}=165.4 \\
T=56 s ; K_{p}=5.9\end{array}$ & $\mu=568.9 \mathrm{~kg}$ & $\begin{array}{c}d v_{\text {wheel }}=0.2 \mathrm{~m} / \mathrm{s} \\
d P_{\text {wheel }}=0.2 \mathrm{~kW}\end{array}$ \\
\hline UF2 & $\begin{array}{c}p_{0}=p_{1}=132.6 \\
T=34 s ; K_{p}=7.0\end{array}$ & $\mu=275.8 \mathrm{~kg}$ & $\begin{array}{c}d v_{\text {wheel }}=0.4 \mathrm{~m} / \mathrm{s} \\
d P_{\text {wheel }}=0.5 \mathrm{~kW}\end{array}$ \\
\hline UF3 & $\begin{array}{c}p_{0}=p_{1}=139.3 \\
T=10 \mathrm{~s} ; K_{p}=2.2\end{array}$ & $\mu=143.9 \mathrm{~kg}$ & $\begin{array}{c}d v_{\text {wheel }}=0.4 \mathrm{~m} / \mathrm{s} \\
d P_{\text {wheel }}=0.5 \mathrm{~kW}\end{array}$ \\
\hline R1 & $\begin{array}{c}p_{0}=p_{1}=134.1 \\
T=38 \mathrm{~s} ; K_{p}=4.7\end{array}$ & $\mu=137.0 \mathrm{~kg}$ & $\begin{array}{c}d v_{\text {wheel }}=0.45 \mathrm{~m} / \mathrm{s} \\
d P_{\text {wheel }}=1 \mathrm{~kW}\end{array}$ \\
\hline R2 & $\begin{array}{c}p_{0}=p_{1}=126.5 \\
T=27 \mathrm{~s} ; K_{p}=1.2\end{array}$ & $\mu=145.7 \mathrm{~kg}$ & $\begin{array}{c}d v_{\text {wheel }}=0.6 \mathrm{~m} / \mathrm{s} \\
d P_{\text {wheel }}=1 \mathrm{~kW}\end{array}$ \\
\hline R3 & $\begin{array}{c}p_{0}=p_{1}=128.0 \\
T=42 s ; K_{p}=2.0\end{array}$ & $\mu=126.2 \mathrm{~kg}$ & $\begin{array}{c}d v_{\text {wheel }}=0.6 \mathrm{~m} / \mathrm{s} \\
d P_{\text {wheel }}=1 \mathrm{~kW}\end{array}$ \\
\hline A1 & $\begin{array}{c}p_{0}=p_{1}=134.1 \\
T=43 s ; K_{p}=1.0\end{array}$ & $\mu=71.9 \mathrm{~kg}$ & $\begin{array}{c}d v_{\text {wheel }}=0.7 \mathrm{~m} / \mathrm{s} \\
d P_{\text {wheel }}=1 \mathrm{~kW}\end{array}$ \\
\hline A2 & $p_{0}=p_{1}=121.6$ & $\mu=99.9 \mathrm{~kg}$ & $\begin{array}{c}d v_{\text {wheel }}=1 \mathrm{~m} / \mathrm{s} \\
d P_{\text {wheel }}=1 \mathrm{~kW}\end{array}$ \\
\hline \hline
\end{tabular}

Fig. 23 represents the fuel saving obtained with the different management strategies and the final battery state of charge. The fuel saving is calculated using the corrected consumption defined in (28).Since PMP and DP have extremely close results, only PMP results are displayed as benchmark. These results are the best minimization performance that the real-time strategies can achieve for the considered cycles, since the setting parameters are adjusted specifically for each cycle. The results confirm the ranking observed in the previous section: A-ECMS performs very well, whereas SDP and OCL are disappointing. The next step is to run a dispersion study with random cycles automatically generated.

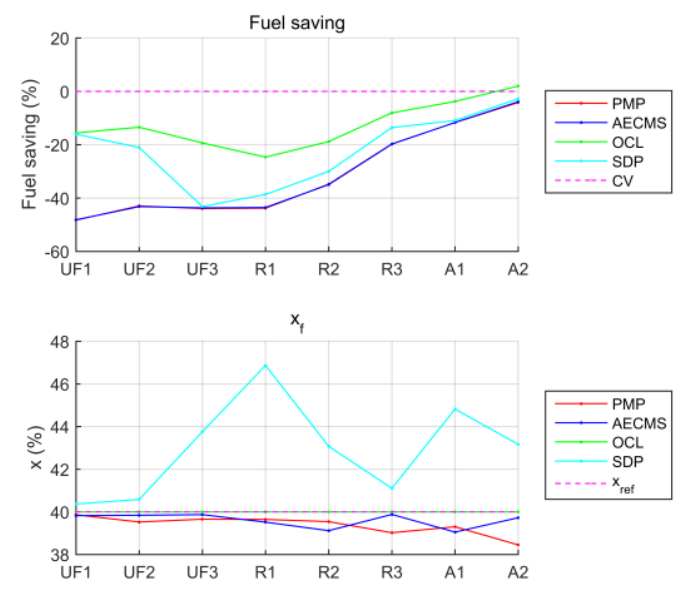

Fig. 23. Real-time strategies' results for INRETS cycles, compared to off-line strategies'. The fuel saving is calculated using the corrected consumption defined in (28).

Fig. 24 shows the highway cycle denoted A2 and an example of random cycle generated by the associated Markov chain model. The speed discretization step is $d v=1 \mathrm{~m} / \mathrm{s}=$ $3.6 \mathrm{~km} / \mathrm{h}$, which appears on the generated cycle, which can have short constant speed periods.
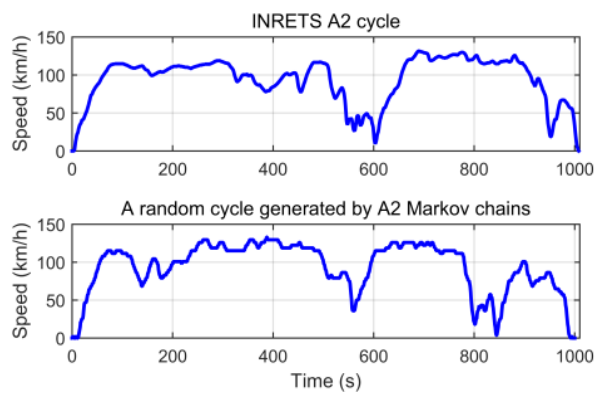

Fig. 24. Top: highway A2 INRETS cycle - Bottom: example of random speed profile produced by the associated Markov chain model

Thousand such cycles have been automatically generated for each of the considered INRETS cycles. Fig. 25 shows the resulting average speed distribution for the 1000 A2-type cycles. This distribution can be characterized by its mean value and standard deviation. These characteristics are plotted for the different types of cycle on Fig. 26, using error bars. It can be observed that the mean speeds are consistent with the data used to produce the random cycles, but it should be noted that due to statistical dispersion a given A1-type random cycle may have a 
lower average speed than a given A2-type. In other words, the $\mathrm{A} 1$ and $\mathrm{A} 2$ average speed distributions overlap.

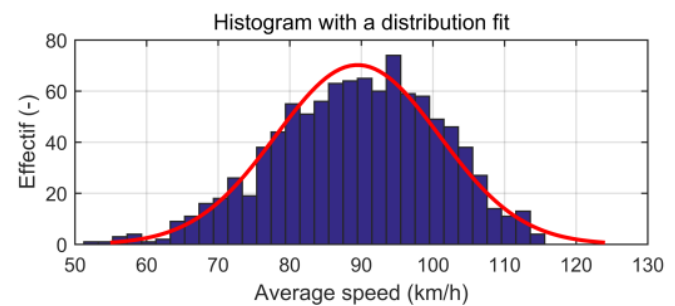

Fig. 25. Average speed of the 1000 random cycles generate by Markov chain model using the A2 cycle data (so-called A2-type random cycle)

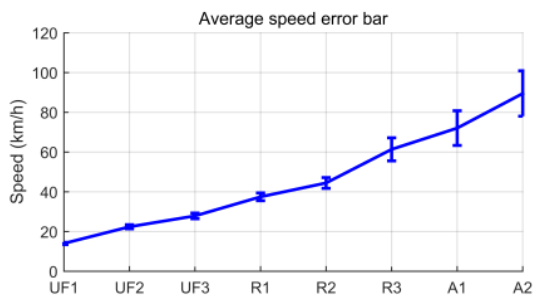

Fig. 26. Statistical characteristics of the 8 series of 1000 random cycles: mean and standard deviation of the average speed of each cycle.

The three real-time strategies and PMP have been applied with the setting parameters given in TABLE IV. For each series of cycles, the mean fuel consumption and standard deviation are plotted in Fig. 27.

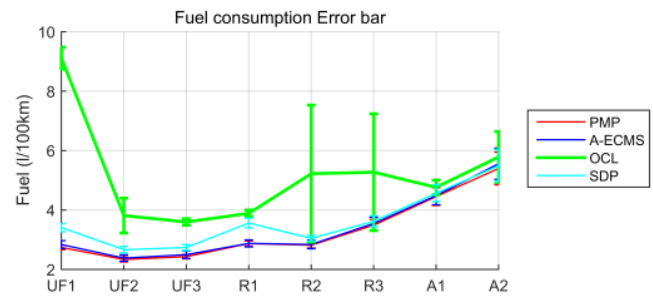

Fig. 27. Mean fuel consumption and standard error for the three real-time strategies. PMP provides reference results.

Obviously, the OCL lacks robustness. In fact, the OCL has a relatively wide interval of parameter $\mu$ inside which the predicted final state of charge remains stable and similar consumptions are found. However, once out of the interval, the OCL becomes instable and results in large fuel consumption usually accompanied by a fully recharged battery state. As the interval depends on the driving cycle, the preset parameter may not suit all the 1000 random cycles. The disappointing performance of OCL method can be explained by the very strong hypothesis it relies on: the ICE consumption is modeled by the average slope of Willans lines (20) and the intercept of Willans lines is not used despite its importance. To confirm this interpretation, we have applied the PMP and OCL strategies to a hybrid powertrain modeled according to OCL assumptions. Results not reported here show that in these conditions, similar performances are obtained. This recently proposed strategy still needs improvement and will not be considered in the rest of the statistical analysis.
Fig. 28 shows the mean and standard deviation of the fuel consumption saving and the corresponding final SOC for PMP, A-ECMS and SDP.
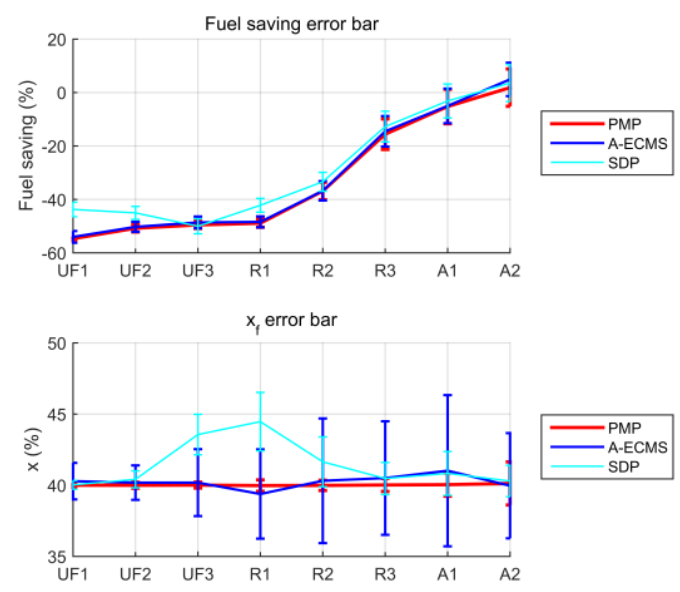

Fig. 28. Top: Mean fuel consumption saving and standard deviation for PMP, A-ECMS and SDP. Bottom: mean final state $x_{f}$ and standard deviation

In all cases, the A-ECMS obtains fuel consumption saving very close to the PMP, which means that one set of parameters specific to each type of driving conditions does help to improve the results of the A-ECMS. Likewise, the SDP's results are promising. The insufficiently fine discretization (cf. TABLE IV) may cause the relatively higher consumption at category UF1, UF2 and R1.

However, the two strategies behave differently on SOC sustaining. As shown in the bottom curve of Fig. 28, the A-ECMS has the biggest variation of the final SOC in category $\mathrm{A} 1$ and the SDP in category R1. What the figure does not tell is that the A-ECMS results in a final SOC between $17.9 \%$ and $60.7 \%$ for the category A1, while the SDP obtains a final SOC between $39.6 \%$ and $49.2 \%$ for R1. It means that even with the same final SOC penalty, SDP is more capable of bringing the SOC back to its reference than A-ECMS.

\section{CONCLUSION}

The present paper provides a complete comparative study of three promising real-time HEV energy management strategies from the literature. Simulation results show that the original OCL requires modifications to achieve good performance on the considered powertrain model. A-ECMS reaches the best fuel saving performance when used with parameter sets adjusted to the type of driving conditions, while SDP better respects the charge sustaining constraint.

During the implementation phase using published material, the important issue of an efficient parameter determination procedure has been tackled. The two off-line methods PMP and DP whose results are generally considered as on-line approach evaluation benchmark have also been investigated to insure their accuracy.

Once well calibrated off-line on the WLTC certification driving cycle, the real-time strategies have been applied to a series of ten driving cycles that represents users' behavior on 
different road trips. Based on those simulation results, the setting parameters of the on-line strategies have been adjusted to each type of cycle and a statistical analysis has then been launched using random cycles generated by a stationary Markov process.

A-ECMS results are sensitive to setting parameters which makes the manual calibration very difficult. The integration of a genetic algorithm did help out with an increasing computational load in return. Moreover, with a wisely chosen parameter set specific to each road type driving conditions, it enables to achieve the maximal fuel saving under any circumstance.

With only one tuning parameter, OCL's calibration phase is much easier than A-ECMS. The simulation results show less sensitive parameter dependence. However this method is not as powerful as A-ECMS because of the very strong ICE modeling hypothesis it relies on. Besides, this method may not be as sensitive as A-ECMS to parameter setting, but only in a limited range of parameter and getting out of it directly leads to false results.

SDP insures the charge-sustaining requirement with a fuel economy performance right behind A-ECMS. As an optimization algorithm based on mathematical models, it achieves the optimal control decision in an average sense. In addition, its quality relies on the accuracy of the probability distribution of the vehicle's driving conditions.

The further work will be

- Developing an on-line driving condition detection mechanism to wisely choose the A-ECMS parameter set;

- Redesigning the OCL method using a more accurate ICE modeling other than the average Willans line slope

- Implementing an infinite horizon SDP [11] instead of the finite horizon one being developed in the present work.

\section{REFERENCES}

[1] F. Odeim, J. Roes, L. Wülbeck, and A. Heinzel, "Power management optimization of fuel cell/battery hybrid vehicles with experimental validation," J. Power Sources, vol. 252, pp.333-343, Apr. 2014.

[2] C. Musardo, G. Rizzoni, and B. Staccia, "A-ECMS: An Adaptive Algorithm for Hybrid Electric Vehicle Energy Management," in Proc. IEEE Conf Decis Control, 2005, pp.1816-1823

[3] S. Onori, L. Serrao, and G. Rizzoni. "Adaptive equivalent consumption minimization strategy for hybrid electric vehicles," in Proc. ASME-DSCC, 2010, pp.499-505.

[4] N. Kim, S. Cha, and H. Peng, "Optimal Control of Hybrid Electric Vehicles Based on Pontryagin's Minimum Principle," IEEE Trans. Control Syst. Technol, vol.19, no.5, pp.1279-1287, Aug. 2011.

[5] S. Kermani, R. Trigui, S. Delprat, B. Jeanneret, and T. M. Guerra, "PHIL Implementation of Energy Management Optimization for a Parallel HEV on a Predefined Route," IEEE Trans. Veh. Technol, vol. 60, no 3, pp.782-792, Mar. 2011.

[6] B. Sampathnarayanan, S. Onori and S. Yurkovich, "An optimal regulation strategy with disturbance rejection for energy management of hybrid electric vehicles," Automatica, vol.50, no.1, pp.128-140, Jan. 2014.

[7] D. Karbowski, N. Kim, and A. Rousseau, "Route-Based Online Energy Management of a PHEV and Sensitivity to Trip Prediction," in IEEE-VPPC, Coimbra, 2014, pp.1-6.

[8] C.-C. Lin, H. Peng, and J. W. Grizzle, "A stochastic control strategy for hybrid electric vehicles," in Proc. Am. Control. Conf, Boston, MA, 2004, pp.4710-4715, vol.5.

[9] L. Johannesson, M. Asbogard, and B. Egardt, "Assessing the Potential of Predictive Control for Hybrid Vehicle Powertrains Using Stochastic Dynamic Programming," IEEE Trans. Intell. Transport. Syst, vol. 8, no. 1, pp. 71-83, March 2007
[10] A. Sciarretta, and L. Guzzella, "Control of hybrid electric vehicles," IEEE Control Syst., vol.27, no.2, pp.60-70, Apr. 2007.

[11] J. Liu, and H. Peng, "Modeling and Control of a Power-Split Hybrid Vehicle," IEEE Trans. Contr. Syst. Technol, vol.16, no.6, pp.1242-1251, Nov. 2008.

[12] S. Bashash, S. J. Moura, J. C. Forman, and H. K. Fathy, "Plug-in hybrid electric vehicle charge pattern optimization for energy cost and battery longevity," J. Power Sources, vol.196, no.1, pp.541-549, Jan. 2011.

[13] S. J. Moura, H. K. Fathy, D. S. Callaway, and J. L. Stein, "A Stochastic Optimal Control Approach for Power Management in Plug-In Hybrid Electric Vehicles," IEEE Trans. Contr. Syst. Technol, vol.19, no.3, pp.545-555, May 2011.

[14] F. Roy, F. Ossart and C. Marchand, "An Optimal Energetic Approach for Systemic Design of Hybrid Powertrain," in IEEE-VPPC, Coimbra, 2014, pp. 1-6.

[15] X. Jiao, and T. Shen, "SDP Policy Iteration-Based Energy Management Strategy Using Traffic Information for Commuter Hybrid Electric Vehicles," Energies, vol.7, no.7, pp. 4648-4675, Jul. 2014.

[16] M. Pourabdollah, N. Murgovski, A. Grauers, and B. Egardt, "Optimal Sizing of a Parallel PHEV Powertrain,” IEEE Trans. Veh. Technol, vol.62, no. 6, pp.2469-2480, Jul. 2013.

[17] D.E.Kirk, "The variational approach to optimal control problems" in Optimal control theory: an introduction, 1st ed. New York: Dover Publications,2004,pp.227-240.

[18] G. Paganelli, G. Ercole, A. Brahma, Y. Guezennec, and G Rizzoniet, "General supervisory control policy for the energy optimization of charge-sustaining hybrid electric vehicles," JSAE review, vol.22, no.4, pp.511-518, Oct. 2001.

[19] D.P.Bertsekas, "The dynamic programming algorithm" in Dynamic programming and optimal control, 3rd ed. Nashua: Athena Scientific, 2005,pp.2-62.

[20] F. H. Clarke, and R. B. Vinter, "The relationship between the maximum principle and dynamic programming," SIAM J. Contr. Optim, vol.25, no.5, pp.1291-1311, 1987.

[21] Q. Jiang, F. Ossart, and C. Marchand, "Real-time HEV energy management strategies", in SGE, Grenoble, Jun.7-9, 2016

[22] M. Tutuianu, P. Bonnel, B. Ciuffo, T. Haniu, N. Ichikawa, A. Marotta, J. Pavlovic, and H. Steven, "Development of the World-wide harmonized Light duty Test Cycle (WLTC) and a possible pathway for its introduction in the European legislation," Transp. Res. Part D Transp. Environ, vol.40, pp.61-75, Oct. 2015.

[23] J.S. Barlow, S. Latham, I.S. McCrae, and P.G. Boulter. (2009 June). A Reference Book of Driving Cycles for use in the measurement of road vehicle [Online]. Available: https://www.gov.uk/government/uploads/system/uploads/attachment_dat a/file/4247/ppr-354.pdf

[24] Q. Gong, Y. Li, and Z-R Peng, "Trip-Based Optimal Power Management of Plug-in Hybrid Electric Vehicles," IEEE Trans. Veh. Technol, vol.57, no.6, pp.3393-3401, Nov. 2008.

[25] G. Souffran, L. Miegeville, and P. Guerin, "Simulation of Real-World Vehicle Missions Using a Stochastic Markov Model for Optimal Powertrain Sizing," IEEE Trans. Veh. Technol, vol.61, no.8, pp.3454-3465, Oct. 2012.

[26] S. Delprat, and T. Hofman, "Hybrid Vehicle Optimal Control: Linear Interpolation and Singular Control," in IEEE-VPPC, Coimbra, 2014, pp.1-6. 


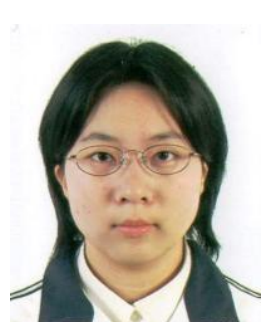

Qi Jiang received the engineering degree from the Institut national des sciences appliquées de Rennes, France, in 2012, in mechanical engineering and automatic control. She is currently pursuing the Ph.D degree in electrical engineering at the Université Paris XI, France. Her current research involves global optimization algorithms and multi-source system energy management.

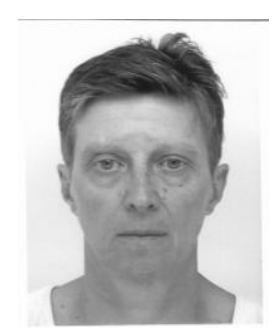

Florence Ossart received the Ph.D. degree in electrical engineering from the Institut National Polytechnique de Grenoble (INPG, France) in 1991 and the HDR degree from the Universite Pierre et Marie Curie (UPMC, France) in 2000. She has worked on magnetic materials modeling and magneto-mechanical coupling in electric machines as a CNRS researcher at the Laboratoire d'Electrotechnique de Grenoble from 1991 to 1997 and at the Laboratoire de Mécanique et Technologie de Cachan from 1997 to 2003. In 2003, she joined the Université Pierre et Marie Curie and the Group of Electrical Engineering of Paris as a full professor. Her current research interests are hybrid electric vehicle energy management and more generally multi-sources multi-loads systems modeling.

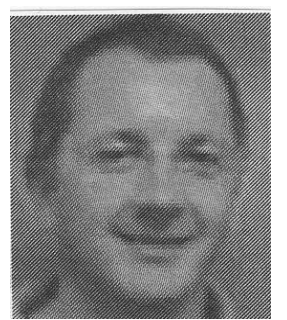

Claude Marchand graduated from the Ecole Normale Supérieure de Cachan, Cachan, France. He receveid the Ph.D. and the HDR degrees from the Université Paris-Sud, Paris, France in 1991 and 1999 respectively. He joined the Université Paris-Sud as an Assistant Professor in 1994 and became a Full Professor in 2000. Since 1988, he has been a researcher at the Laboratoire de Génie Electrique de Paris (LGEP), where his research interests are in eddy current nondestructive testing and in the design and control of electrical actuators. Since 2015, he is head of the Group of Electrical Engineering of Paris (GeePs), a new laboratory created by merging the LGEP and the research activities of the "Energy Department" of CentraleSupelec. 